\title{
INVESTIGACIONES
}

\section{Los Procesos de Evaluación y sus Consecuencias. Análisis de la Experiencia del Profesorado de Educación Física*}

\author{
Assessment Processes and their Consequences: \\ An Analysis of the Physical Education Teacher's Experience
}

\author{
Miriam Sonlleva ${ }^{a}$, Suyapa Martínez, Roberto Monjas ${ }^{c}$ \\ a Universidad de Valladolid \\ Correo electrónico: miriam.sonlleva@uva.es \\ ${ }^{\mathrm{b}}$ Universidad de Valladolid \\ Correo electrónico: suyapa@pdg.uva.es. \\ ${ }^{\mathrm{c}}$ Universidad de Valladolid \\ Correo electrónico: rmonjas@mpc.uva.es.
}

\section{RESUMEN}

La evaluación supone uno de los momentos más controvertidos de la formación inicial de los docentes de Educación Física (EF). El estudio que presentamos trata de analizar sus vivencias ante este hecho, a través del análisis de los sentimientos y emociones que genera la evaluación entre los estudiantes, las interacciones entre profesores y alumnos en el proceso de evaluación y los aspectos de la evaluación mejor valorados. Para ello, utilizamos una encuesta, realizada a 491 profesores de 17 universidades españolas, que se completa con 12 entrevistas personales. Los resultados nos permiten afirmar que los profesionales de EF sienten ansiedad y temor hacia la evaluación; consideran fundamental la relación entre profesores y estudiantes para mejorar su proceso formativo; y afirman que el conocimiento previo del sistema de evaluación y su acuerdo son aspectos básicos para la motivación del aprendizaje y la reducción de los síntomas de tensión.

Palabras claves: educación superior, evaluación formativa, experiencias de docentes, proceso de aprendizaje

\begin{abstract}
Assessment entails one of the most contentious moments in the initial formation of physical education (PE) teachers. This study attempts to analyze teacher experiences in light of this fact through an analysis of the feelings and emotions generated among students by assessment, the interactions between teachers and students in the process, and the highest-rated aspects of assessment. For this purpose, we conducted a survey of 491 teachers from 17 Spanish universities, the results of which are complemented by 12 personal interviews. Based on the data gathered, we suggest that PE professionals feel anxiety and fear toward assessment; they consider the relationship between teachers and students fundamental in improving their training process; and they affirm that prior knowledge of the assessment system and its arrangement are fundamental aspects for motivation in learning and the reduction of stress.
\end{abstract}

Keywords: higher education, formative assessment evaluation, teacher experience, learning process.

Este estudio se ha llevado a cabo dentro del proyecto de I+D+i: "Las competencias docentes en la formación inicial del profesorado de educación física". Convocatoria de noviembre de 2013 del Programa Estatal de Investigación, Desarrollo e Innovación Orientada a los Retos de la Sociedad, en el marco del Plan Estatal de Investigación Científica y Técnica y de Innovación 2013-2016. Referencia: EDU 2013-42024-R. Duración: 3 años (2014-2016). 
Estudios Pedagógicos XLIV, $\mathrm{N}^{\circ}$ 2: 329-351, 2018

LOS PROCESOS DE EVALUACIÓN Y SUS CONSECUENCIAS. ANÁLISIS DE LA EXPERIENCIA DEL PROFESORADO DE EDUCACIÓN FÍSICA

\section{INTRODUCCIÓN}

La evaluación supone una de las dimensiones docentes que presenta mayores controversias tanto para los profesores universitarios como para los maestros en formación y los egresados (Flórez, 1999; Palacios \& López-Pastor, 2013). Los primeros dicen no sentirse suficientemente formados en esta tarea docente (Flórez, 1999; Nieto, 2000; Trillo, 2005; Zabalza, 2003); y reproducen en las aulas los modelos tradicionales de evaluación, que ellos mismos vivieron siendo alumnos (López-Pastor, 2004; Tejedor, 1998). Los maestros en formación y los egresados muestran su descontento porque consideran que la evaluación es una forma de control que ejercen los profesores sobre el alumnado, que se reduce en exclusiva a los resultados, con independencia de cómo han sido estos conseguidos y que no se sienten partícipes de las pruebas de evaluación, ni en cuanto al acuerdo de las fechas ni en relación al sistema de calificación (Trillo \& Porto, 1999).

Los estudios realizados hasta el momento acerca de esta problemática apuntan que una forma de resolverla, con probados resultados positivos, es a través de procesos de evaluación formativa y compartida. Este tipo de evaluación permite mejorar y optimizar los procesos de enseñanza aprendizaje (Brown \& Glasner, 2003; López-Pastor, 2009) y ayuda a los estudiantes a la mejora de los resultados de aprendizaje y de sus competencias profesionales (López-Pastor, 2008; Martínez, Santos \& Castejón, 2017). La participación del alumno en su propio proceso de aprendizaje y en su evaluación, la calificación dialogada, la negociación curricular y la interacción entre estudiantes y profesores (Brown \& Glasner, 2003; LópezPastor, 2009; Rodríguez, Ibarra \& García, 2013; Silva \& López, 2015) son aspectos que permiten que el docente en formación sea consciente del trabajo que realiza, reflexione sobre su labor y refuerce su motivación personal para el aprendizaje y el logro (Porto, 2009).

Una de las líneas de trabajo desde las que se puede investigar el sistema de evaluación universitario en la formación inicial del profesorado (FIP) es a través de la percepción que los estudiantes tienen sobre cómo son evaluados. Publicaciones como las de Buskist, Sikorski, Buckley y Saville (2002), Casillas (2006), Gargallo et al. (2010), Hortigüela \& Pérez-Pueyo (2016), revelan la importancia que tiene conocer esta valoración del alumno, ya que permite reconducir el proceso de evaluación y mejorarlo (Ricoy \& FernándezRodríguez, 2013).

El trabajo que presentamos tiene como punto de partida la premisa que apunta que "la evaluación no es algo que se hace a los estudiantes. Es una actividad hecha por los estudiantes" (Brown \& Glasner, 2007, p. 189). De este modo, no entendemos una investigación sobre la evaluación sin incluir la voz de los estudiantes. No son abundantes los estudios que tratan de profundizar en estas percepciones y vivencias sobre la evaluación que los docentes han experimentado en su formación inicial (Hamodi \& López-Pastor, 2012; Hortigüela, Abella \& Pérez-Pueyo, 2015a; López-Pastor \& Palacios, 2012; Ricoy \& Fernández-Rodríguez, 2013; Silva \& López-Pastor, 2015; Trillo \& Porto, 1999) y la mayoría de los estudios tratan de abordarla desde metodologías cuantitativas, donde la opinión del estudiante se limita a una respuesta numérica.

López-Pastor y Palacios (2012) plantean que para la mejora de este tipo de estudios resulta necesario el uso de metodologías cualitativas, pues el análisis interpretativo de datos mejoraría los resultados y conclusiones obtenidos hasta el momento. Tímidamente han ido apareciendo trabajos que tratan de paliar estas carencias a través de estudios mixtos o estudios de caso (Angelini, 2016; Herranz \& López-Pastor, 2017; Ricoy \& Fernández-Rodríguez, 2013). 
La investigación que presentamos intenta progresar en esta laguna del conocimiento, tratando de conocer, desde una metodología mixta, cómo han vivido los docentes de Educación Física la evaluación durante su formación inicial como maestros. Para el desarrollo de este objetivo, profundizaremos en tres temas específicos, poco analizados hasta el momento: sentimientos y emociones que genera la evaluación entre los estudiantes; influencia de las interacciones entre docentes y estudiantes en la concepción de la evaluación; y aspectos mejor valorados por los estudiantes para la evaluación de sus aprendizajes.

\section{METODOLOGÍA}

\subsection{FASES DE LA INVESTIGACIÓN}

El estudio que presentamos parte de una metodología de investigación mixta, que ha sido llevada a cabo en cuatro etapas de trabajo diferentes. Inicialmente, se elaboró un cuestionario sobre vivencias en torno a la evaluación y se caracterizó a los sujetos que participarían en la muestra. En una segunda fase se procedió a la recogida de información y posterior tratamiento de los datos cuantitativos, a través de un análisis descriptivo. La administración de cuestionarios se realizó por parte del equipo de investigación, durante el curso académico 2014-2015. Posteriormente, se elaboró un guion de entrevista cerrado y se realizaron entrevistas individuales a 12 participantes, correspondientes en edad con el mayor volumen de la muestra. Las entrevistas fueron realizadas por los autores del trabajo, entre los meses de noviembre y diciembre del año 2017. La tercera etapa concluyó con el análisis cualitativo de los datos. Finalmente, procedimos a la redacción del informe que aquí presentamos, a través de la discusión conjunta de los datos obtenidos.

\subsection{PARTICIPANTES}

El estudio ha sido realizado con un total de 491 docentes (58,9\% hombres y 41,1\% mujeres), que finalizaron sus estudios entre los años 2000 y 2015. La mayoría de los encuestados tiene una edad inferior a 30 años $(91,7 \%)$ y fueron estudiantes de las Facultades de Ciencias de la Actividad Física y el Deporte (61\%) y las Facultades de Educación (39\%), de las localidades de Albacete, Barcelona, Córdoba, Huesca, La Laguna, León, Lleida, Madrid, Murcia, Segovia, Valencia, Valladolid, Vitoria y Zamora (Tabla 1).

Tabla 1. Distribución de la muestra por localidades

\begin{tabular}{|l|c|c|c|c|}
\hline & Frecuencia & Porcentaje & Porcentaje válido & Porcentaje acumulado \\
\hline Segovia & 21 & 4,3 & 4,4 & 4,4 \\
\hline Albacete & 62 & 12,6 & 12,9 & 17,3 \\
\hline Granada & 1 & 0,2 & 0,2 & 17,5 \\
\hline Madrid & 6 & 1,2 & 1,3 & 18,8 \\
\hline Valencia & 6 & 1,2 & 1,3 & 20,0 \\
\hline
\end{tabular}




\begin{tabular}{|l|c|c|c|c|}
\hline Valladolid & 25 & 5,1 & 5,2 & 25,3 \\
\hline Barcelona & 66 & 13,4 & 13,8 & 39,0 \\
\hline Vitoria & 30 & 6,1 & 6,3 & 45,3 \\
\hline Córdoba & 24 & 4,9 & 5,0 & 50,3 \\
\hline León & 43 & 8,8 & 9,0 & 59,3 \\
\hline Huesca & 59 & 12,0 & 12,3 & 71,6 \\
\hline La Laguna & 8 & 1,6 & 1,7 & 73,3 \\
\hline Lleida & 60 & 12,2 & 12,5 & 85,8 \\
\hline Murcia- UCAM & 49 & 10,0 & 10,2 & 96,0 \\
\hline Zamora & 15 & 3,1 & 3,1 & 99,2 \\
\hline Zaragoza & 2 & 0,4 & 0,4 & 99,6 \\
\hline Murcia - UMU & 2 & 0,4 & 0,4 & 100,0 \\
\hline Total & 479 & 97,6 & 100,0 & \\
\hline Perdidos sistema & 12 & 2,4 & & \\
\hline Total & 491 & 100,0 & & \\
\hline
\end{tabular}

\subsection{INSTRUMENTOS PARA LA RECOGIDA DE DATOS}

Como explicábamos anteriormente, la toma de datos se realizó mediante dos instrumentos: un cuestionario (Anexo 1) y una entrevista cerrada (Anexo 2).

En relación al cuestionario, para recoger la valoración que los 491 docentes hacen de la evaluación se siguió un procedimiento de construcción y validación de este instrumento acorde con los criterios Thurstone y Likert. La presentación final del cuestionario consiste en una escala tipo Likert de cinco puntos (0-5), donde "0" es "nada de acuerdo", "1" es "poco de acuerdo", "2" es "ni acuerdo ni desacuerdo", "3" es "de acuerdo" y "4" es "muy de acuerdo". Con el objetivo de garantizar que el instrumento cumpliera con los requisitos de calidad suficientes, los pasos que se siguieron en su elaboración y diseño fueron: análisis documental (para identificar las variables más relevantes objeto de estudio, así como los instrumentos aplicados en investigaciones similares); elaboración de un conjunto inicial de ítems (de acuerdo a los datos descubiertos en el análisis documental); valoración de la pertinencia y relevancia del conjunto inicial de ítems (a través de una prueba de expertos); y estudio piloto (aplicado a una muestra de egresados de características similares a la muestra definitiva). Elaborada la escala definitiva se procedió a su aplicación, analizando sus características psicométricas, en cuanto a la validez de contenido del instrumento y su fiabilidad. El coeficiente calculado para la escala final de 7 ítems resultó ser satisfactorio ( $\alpha$ de Cronbach 0.866).

Este instrumento fue complementado con una entrevista cerrada, que se realizó a 12 personas de la muestra ( 7 hombres y 5 mujeres), que tienen una edad media de 30 años. Para elaborar el guion de la entrevista se procedió a reformular las siete preguntas del cuestionario, para que el participante pudiera expresar de forma abierta su opinión. El objetivo de este instrumento es recuperar el mayor volumen de información específica posible y aportar algunos datos significativos que el cuestionario no permite analizar. 
Tanto el cuestionario como la entrevista tienen un carácter anónimo y en la redacción del trabajo final hemos intentado proteger la confidencialidad de los datos personales de los participantes, eliminando aquellos aspectos de la experiencia que pudieran permitir reconocer a los protagonistas del estudio.

\subsection{ANÁLISIS DE LOS DATOS}

Los datos cuantitativos fueron analizados mediante el paquete estadístico SPSS 20.0. y en cada uno de los siete ítems de la encuesta, se ha profundizado en el análisis de las variables: sexo, tipo de estudios y años de experiencia docente. Puesto que el objetivo de la investigación es indagar sobre las vivencias de los docentes en torno a la evaluación se ha trabajado fundamentalmente con las frecuencias relativas de cada una de las posibles respuestas y los porcentajes. Además, se han realizado pruebas de medias simples e independientes en los datos de cada categoría de análisis.

Los datos cualitativos, recogidos en las entrevistas individuales, han sido categorizados a través del programa ATLAS.ti, recurriendo como guía para su análisis a los objetivos que marcan el estudio. El código que utilizaremos para presentar las citas literales será: sexo, edad, estudios. Para el sexo utilizaremos las siglas H (hombres), M (mujeres), para los estudios recurriremos a las siglas EMEF (Estudiantes de Magisterio de Educación Física) y ECAFID (Estudiantes de Ciencias de la Actividad Física y el Deporte).

La presentación de los resultados se realizará a través de tres categorías de análisis, coincidentes con los objetivos específicos que señala la investigación: sentimientos y emociones que produce la evaluación en los estudiantes; influencia de las interacciones entre docentes y estudiantes en la concepción de la evaluación; y aspectos mejor valorados por los estudiantes para la evaluación de sus aprendizajes.

\section{ANÁLISIS DE RESULTADOS}

\subsection{SENTIMIENTOS Y EMOCIONES QUE PRODUCE LA EVALUACIÓN EN LOS ESTUDIANTES}

\subsubsection{La recogida de información para la evaluación: ansiedad, tensión y nerviosismo}

El análisis de los datos cuantitativos realizado a la muestra nos permite apreciar que los participantes no consideran que la recogida de información para la evaluación genere un alto grado tensión y nerviosismo (Tabla 2). Más de un 30\% de los encuestados no expresa ni acuerdo ni desacuerdo con la afirmación.

Tabla 2. Media y dt de los ítems 1, 2 y 3 correspondientes a sentimientos que produce la evaluación

\begin{tabular}{|l|c|c|}
\hline Ítem & $\mathrm{M}$ & $d t$ \\
\hline 1. La recogida de información para la evaluación nos genera tensión y nerviosismo. & 2,52 & 1,082 \\
\hline 2. La recogida de información para la evaluación fomenta la motivación para el aprendizaje. & 2,32 & 1,075 \\
\hline 3. La evaluación positiva repercute en la autoestima del/a estudiante. & 3,46 &, 687 \\
\hline
\end{tabular}


Las entrevistas arrojan algunos datos para profundizar en el conocimiento de esta incertidumbre que los docentes muestran en sus respuestas. Los participantes afirman que las situaciones vividas individualmente, en las diferentes asignaturas de la FIP y las respectivas vivencias con el profesorado de cada materia no les permiten tener una respuesta unívoca ante esta pregunta.

Cuando pienso en el momento de recogida de información me vienen dos cosas a la cabeza: el instrumento y el profesor. Si hablamos de trabajos en clase, no siento ninguna tensión, pero si de lo que se trata es de exámenes, sí. También me ponía muy nerviosa la observación, principalmente si esta venía del profesor. (H, 30, EMEF).

Dentro del grupo de estudiantes de EF, las mujeres muestran mayores niveles de ansiedad que los hombres. La prueba de diferencia de medias realizada nos advierte que las diferencias entre sexos resultan significativas (Tabla 3 ).

Tabla 3. Media, $d t$ y diferencia de medias de los ítems 1,2 y 3 , por sexo

\begin{tabular}{|c|c|c|c|c|c|c|}
\hline Ítem & $S$ & M & $d t$ & $\mathrm{U}$ & Z & Sig* \\
\hline \multirow{2}{*}{$\begin{array}{l}\text { 1. La recogida de información para la } \\
\text { evaluación nos genera tensión y nerviosismo. }\end{array}$} & $\mathrm{H}$ & 2,38 & 1,096 & \multirow{2}{*}{23066,000} & \multirow{2}{*}{$-3,573$} & \multirow{2}{*}{,000 } \\
\hline & M & 2,73 & 1,038 & & & \\
\hline \multirow{2}{*}{$\begin{array}{l}\text { 2. La recogida de información para la } \\
\text { evaluación fomenta la motivación para el } \\
\text { aprendizaje. }\end{array}$} & $\mathrm{H}$ & 2,35 & 1,036 & \multirow{2}{*}{27197,500} & \multirow{2}{*}{,- 413} & \multirow{2}{*}{,680 } \\
\hline & M & 2,28 & 1,128 & & & \\
\hline \multirow{2}{*}{$\begin{array}{l}\text { 3. La evaluación positiva repercute en la } \\
\text { autoestima del/a estudiante. }\end{array}$} & $\mathrm{H}$ & 3,39 & ,736 & \multirow{2}{*}{24640,000} & \multirow{2}{*}{$-2,315$} & \multirow{2}{*}{,021 } \\
\hline & M & 3,55 & 601 & & & \\
\hline
\end{tabular}

$\mathrm{S}=\operatorname{sexo}(\mathrm{H}=$ hombres. $\mathrm{M}=$ mujeres $) . \mathrm{U}=\mathrm{U}$ de Mann Whitney. $* p \leq .05$

Los relatos de las participantes ante este hecho aportan el fundamento de estas diferencias, pues las docentes explican que la observación del profesor durante alguna práctica, el momento anterior y posterior a la realización de un examen o las exposiciones en clase de un trabajo, les produce angustia y temor. Los hombres, a pesar de explicar que la recogida de información para la evaluación les genera tensión, no detallan qué realidades vividas en su FIP les hacen llegar a tener ese tipo de sentimientos.

Me ponía nerviosa cuando tenía que hacer un examen y luego, hasta que no te daban la nota, tenía los nervios presentes y... lo que también he llevado siempre mal son las exposiciones. Recuerdo una de las primeras veces que teníamos que exponer un proyecto en clase y a mí me imponía mucho que la mayoría de los compañeros que me escuchaban fueran chicos... me daba una vergüenza enorme. (M, 30, EMEF).

Los estudios realizados por los participantes también aportan algunas diferencias entre grupos. Los maestros de EF expresan niveles de ansiedad mayores ante las pruebas 
de evaluación, que los de CAFID-INEF. La prueba de medias realizada presenta valores significativos (Tabla 4).

Tabla 4. Media, $d t$ y diferencia de medias de los ítems 1, 2 y 3 por titulación

\begin{tabular}{|c|c|c|c|c|c|c|}
\hline Ítem & $\mathrm{T}$ & M & $d t$ & $\mathrm{U}$ & $\mathrm{Z}$ & Sig* \\
\hline \multirow{2}{*}{$\begin{array}{l}\text { 1. La recogida de información para la evaluación } \\
\text { nos genera tensión y nerviosismo. }\end{array}$} & A & 2,65 & 1,116 & \multirow{2}{*}{24498,500} & \multirow{2}{*}{$-2,370$} & \multirow{2}{*}{,018 } \\
\hline & B & 2,44 & 1,059 & & & \\
\hline \multirow{2}{*}{$\begin{array}{l}\text { 2. La recogida de información para la evaluación } \\
\text { fomenta la motivación para el aprendizaje. }\end{array}$} & A & 2,32 & 1,151 & \multirow{2}{*}{27372,000} & \multirow{2}{*}{,- 017} & \multirow{2}{*}{,987 } \\
\hline & B & 2,32 & 1,027 & & & \\
\hline \multirow{2}{*}{$\begin{array}{l}\text { 3. La evaluación positiva repercute en la } \\
\text { autoestima del/a estudiante. }\end{array}$} & A & 3,49 & ,625 & \multirow{2}{*}{26479,000} & \multirow{2}{*}{,- 668} & \multirow{2}{*}{, 504} \\
\hline & B & 3,43 & ,727 & & & \\
\hline
\end{tabular}

$\mathrm{T}=$ titulación $(\mathrm{A}=$ Magisterio EF. $\mathrm{B}=\mathrm{CAFID}-\mathrm{INEF}) . \mathrm{U}=\mathrm{U}$ de Mann Whitney. * $p \leq .05$

Los datos recogidos en las entrevistas descubren el porqué de estas diferencias, ya que mientras los maestros de EF consideran que la calificación supone un hecho destacable en su FIP y lo viven con cierto agobio, los de CAFID-INEF expresan que están familiarizados con esta realidad y la viven de forma menos perturbadora.

El alumnado suele tener una visión de la evaluación como únicamente de calificación, de suspenso o aprobado. Por ello, siempre que hablamos de temas relacionados a la evaluación, por dentro surge la idea de calificar. (H, 28, EMEF).

La experiencia de los docentes resulta ser otro de los aspectos relevantes para poder conocer diferencias entre los grupos de sujetos que participan en la muestra (Tabla 5). Los datos recogidos señalan que aquellos participantes sin experiencia presentan índices medios de ansiedad $(50,2 \%)$ ante la recogida de información para la evaluación. Este hecho va aumentando cuando los maestros comienzan sus primeras experiencias en el aula, llegando a ser el momento álgido entre los tres y los cuatro años de su presencia en los centros, donde un $76 \%$ de los encuestados dice sentir alta o muy alta tensión hacia las pruebas de evaluación. Después del cuarto año, los niveles descienden de nuevo. Las diferencias entre medias resultan significativas entre este nivel de experiencia y el resto de grupos. 
Estudios Pedagógicos XLIV, $\mathrm{N}^{\circ}$ 2: 329-351, 2018

LOS PROCESOS DE EVALUACIÓN Y SUS CONSECUENCIAS. ANÁLISIS DE LA EXPERIENCIA DEL PROFESORADO DE EDUCACIÓN FÍSICA

Tabla 5. Media, $d t$ y prueba de medias de los ítems 1,2 y 3 , por años de experiencia

\begin{tabular}{|c|c|c|c|c|c|c|}
\hline Ítem & $\mathrm{E}$ & M & $d t$ & Chi-cuadrado & gl & Sig* \\
\hline \multirow{4}{*}{$\begin{array}{l}\text { 1. La recogida de información para la evaluación } \\
\text { nos genera tensión y nerviosismo. }\end{array}$} & A & 2,48 & 1,073 & \multirow{4}{*}{9,201} & \multirow{4}{*}{3} & \multirow{4}{*}{,027 } \\
\hline & B & 2,64 & 1,28 & & & \\
\hline & $\mathrm{C}$ & 3,10 & ,768 & & & \\
\hline & $\mathrm{D}$ & 2,31 &, 855 & & & \\
\hline \multirow{4}{*}{$\begin{array}{l}\text { 2. La recogida de información para la evaluación } \\
\text { fomenta la motivación para el aprendizaje. }\end{array}$} & A & 2,33 & 1,048 & \multirow{4}{*}{0,33} & \multirow{4}{*}{3} & \multirow{4}{*}{,998 } \\
\hline & $\mathrm{B}$ & 2,32 & 1,205 & & & \\
\hline & $\mathrm{C}$ & 2,30 & 1,129 & & & \\
\hline & $\mathrm{D}$ & 2,25 & 1,215 & & & \\
\hline \multirow{4}{*}{$\begin{array}{l}\text { 3. La evaluación positiva repercute en la } \\
\text { autoestima del/a estudiante. }\end{array}$} & $\mathrm{A}$ & 3,47 &, 674 & \multirow{4}{*}{3,530} & \multirow{4}{*}{3} & \multirow{4}{*}{,317 } \\
\hline & $\mathrm{B}$ & 3,34 & ,750 & & & \\
\hline & $\mathrm{C}$ & 3,57 & ,746 & & & \\
\hline & $\mathrm{D}$ & 3,38 & 650 & & & \\
\hline
\end{tabular}

$\mathrm{E}=$ experiencia $(\mathrm{A}=\sin$ experiencia. $\mathrm{B}=1-2$ años. $\mathrm{C}=3-4$ años. $\mathrm{D}=+5$ años $)$. Prueba de Kruskal-Wallis * $p \leq .05$

Cuando conversamos con los docentes sobre este hecho, los datos cualitativos presentan semejantes resultados. Con sus narraciones descubrimos que los primeros años como maestros son vividos por los participantes con algunas tensiones iniciales ante la novedad de la docencia y el sistema de evaluación. Sin embargo, a partir de los cuatro o cinco años en ejercicio, comienzan a sentirse más seguros de su labor, a dejar de recurrir a prácticas pedagógicas de evaluación interiorizadas en su etapa como alumnos y a experimentar nuevos modelos de evaluación con sus estudiantes.

\subsubsection{La motivación a examen}

La motivación por las pruebas de evaluación resulta ser otro de los aspectos peor valorados por los participantes. Más de la mitad $(50,6 \%)$ no considera que la recogida de información para la evaluación fomente la motivación para el aprendizaje (Tabla 2). El análisis cualitativo no aporta datos relevantes ante esta pregunta, que es contestada por los protagonistas de forma sucinta. Los participantes afirman que ni en la FIP ni durante su posterior carrera como docentes, han tenido la sensación de que la evaluación ayude a motivar al estudiante para mejorar sus conocimientos y destrezas.

Considero que el alumnado apenas es consciente de qué le puede aportar. (H, 28, EMEF).

Esta respuesta es mantenida tanto por hombres como por mujeres, sin apreciar ninguna diferencia significativa (Tabla 3). Ambos grupos de personas explican en sus entrevistas 
que la mayoría de sus pruebas de evaluación durante los años de carrera no tenían ningún tipo de retroalimentación y eran utilizadas para informar al profesor sobre el conocimiento que tenían los estudiantes sobre un tema concreto. Esta experiencia previa es la que no les permite reconocer que la evaluación puede convertirse en un proceso de aprendizaje.

En la mayoría de las asignaturas, los profesores recurrían a un examen, que contaba más de la mitad de la nota. Sólo podías saber qué habías hecho mal, si ibas a la revisión, pero no había posibilidad de mejorar el resultado. (M, 30 años, EMEF).

Las frecuencias de respuesta se repiten cuando las analizamos en relación con el tipo de estudios que los participantes han cursado. Tanto los antiguos estudiantes de Magisterio de EF como los de CAFID-INEF expresan haber sentido poca motivación para el aprendizaje durante sus procesos de evaluación, no existiendo diferencias significativas entre las respuestas de ambos grupos de docentes (Tabla 4). Obtener buenas calificaciones y superar las asignaturas son las contestaciones más repetidas en el análisis de datos cualitativos, siendo solo dos las participantes que, tras haber vivido procesos de evaluación formativa en varias asignaturas de su FIP, explican haber sentido algún tipo de motivación para aprender en sus evaluaciones.

La única motivación que me provocaba la recogida de información era sacar buena nota. (H, 29, ECAFID).

En relación con la experiencia, tampoco existen diferencias significativas (Tabla 5). Los análisis muestran cómo los docentes de EF parecen estar de acuerdo, independientemente de los años que lleven ejerciendo la profesión, en que la recogida de información no ayuda a mejorar el aprendizaje ni le influye. Estos mismos datos los encontramos cuando ahondamos en las entrevistas de los participantes.

Desde luego, no han sido las pruebas de evaluación lo que me ha motivado a aprender. Era algo que sabía que tenía que hacer y ya está. (H, 26, ECAFID).

\subsubsection{Repercusiones de la evaluación en la autoestima del estudiante}

Los encuestados exponen en un alto porcentaje $(91,1 \%)$ estar de acuerdo o muy de acuerdo con que la evaluación positiva de los aprendizajes repercute de forma directa en la autoestima del estudiante (Tabla 2). Las entrevistas proyectan los mismos resultados que el análisis cuantitativo. Los doce docentes entrevistados dan cuenta de cómo una evaluación positiva en su FIP les ha permitido sentirse más seguros, estar más motivados y presentar mayor predisposición hacia el aprendizaje.

Te motiva a seguir trabajando y te hace sentir que estás siguiendo el camino adecuado de cara a conseguir un mayor aprendizaje. (M, 39, EMEF).

Hombres y mujeres parecen estar de acuerdo en esta repercusión, no encontrando diferencias significativas entre ambos grupos (Tabla 3). A partir de los datos cualitativos vemos cómo los docentes explican que la evaluación positiva de sus aprendizajes ha mejorado su autoestima y su autoconcepto, hecho que es aún más destacable en el grupo 
de mujeres entrevistadas. Pero los datos cualitativos no solo explican cómo se sienten los participantes ante la evaluación positiva, sino que también dan muestras de una crítica velada hacia cómo es vivida la evaluación negativa.

El profesorado suele centrarse únicamente en los aspectos negativos como un intento porque mejoren. Sin embargo, se olvidan de la importancia de señalar también los aspectos positivos para asentar esos aprendizajes y favorecer aspectos como la autoestima. (H, 28, EMEF).

Tanto los maestros de EF como los profesores de CAFID-INEF, afirman estar de acuerdo o muy de acuerdo con la relación entre evaluación positiva y mejora de la autoestima (Tabla 4). Estos mismos niveles se presentan cuando analizamos los datos según los años de experiencia, estando todos los grupos contenidos en medias entre los 3,4 y los 3,6 grados de acuerdo, independientemente de los años de experiencia docente de los participantes, no encontrando diferencias significativas entre grupos (Tabla 5).

Desde los datos cualitativos, los participantes siguen explicando sus experiencias en relación con las calificaciones negativas. Los docentes afirman que las malas notas, recibidas sin ningún tipo de retroalimentación, les hicieron sentir en su formación inicial, que no servían para la tarea docente, llegándose a cuestionar su vocación hacia el magisterio.

Reiteradas evaluaciones negativas pueden mermar el autoconcepto académico del estudiante, bajando su autoestima personal al no creerse eficaz en esa faceta de su vida. Se provoca así un círculo de fracaso del que es muy difícil salir. (M, 24, EMEF).

\subsection{INFLUENCIA DE LA INTERACCIÓN ENTRE DOCENTES Y ESTUDIANTES EN LAS VIVENCIAS SOBRE LA EVALUACIÓN}

En los recuerdos que los protagonistas tienen de la evaluación, ocupa un papel central el profesor. La interacción entre esta figura y los estudiantes resulta ser fundamental para la mejora del proceso de evaluación (Tabla 6).

Tabla 6. Media y dt del ítem 4, correspondiente a interacción entre docentes y estudiantes

\begin{tabular}{|l|c|c|}
\hline Ítem & M & $d t$ \\
\hline 4. La interacción entre profesores y estudiantes favorece el proceso de evaluación. & 3,51 &, 750 \\
\hline
\end{tabular}

Los participantes entrevistados revelan que el diálogo entre profesores y alumnos; el conocimiento que el educador tenía de ellos y ellas (tanto a nivel académico como personal); y la actitud que los docentes mostraron hacia los estudiantes en el aula, condicionó en gran medida sus experiencias en torno a la evaluación.

Las experiencias que viví en mi formación inicial hacen que hoy me resulte difícil comprender un proceso de evaluación tradicional. Si no me preocupo por conocer al 
alumno, si no sé qué cualidades y potencialidades tiene, si sólo me centro en los resultados que ha tenido en una prueba y no en su evolución... ¿de qué le servirá la evaluación?. (H, 30, ECAFID).

Hombres y mujeres entienden que esta relación es crucial si se quiere llevar a cabo una verdadera evaluación formativa, no existiendo diferencias significativas entre sexos (Tabla 7).

Tabla 7. Media, $d t$ y diferencia de medias del ítem 4, por sexos

\begin{tabular}{|l|c|c|c|c|c|}
\hline & $\mathrm{M}$ & $d t$ & $\mathrm{U}$ & $\mathrm{Z}$ & Sig* $^{*}$ \\
\hline Hombre & 3,47 &, 0810 & 27109,000 &,- 793 &, 428 \\
\hline Mujer & 3,57 &, 649 & \multicolumn{3}{|l}{} \\
\hline
\end{tabular}

$\mathrm{U}=\mathrm{U}$ de Mann Whitney. ${ }^{*} p \leq .05$

Con las palabras de los protagonistas, vemos que los modos ideales de establecer este tipo de interacciones son diferentes para hombres que para mujeres. Los docentes masculinos no hacen visibles sus prioridades, o hablan del diálogo como forma de establecer este contacto; las mujeres sí mencionan algunas de las características que consideran importantes en este tipo de relaciones. La empatía, la ayuda o la comprensión son algunos rasgos que ellas destacan en las relaciones entre docentes y estudiantes y que aplican en su trayectoria docente.

La interacción facilita el diálogo igualitario de forma que se fomenta el aprendizaje al poder emitir y recibir críticas constructivas de forma más empática. (M, 39, EMEF).

Cuando realizamos un análisis de datos con relación a los estudios realizados por los participantes, tampoco encontramos diferencias significativas (Tabla 8). Tanto los antiguos estudiantes en Magisterio de EF, como los de CAFID-INEF, consideran alta o muy alta la importancia de este tipo de relaciones para mejorar los aprendizajes.

Tabla 8. Media, $d t$ y diferencia de medias del ítem 4, por titulación

\begin{tabular}{|l|c|c|c|c|c|}
\hline & $\mathrm{M}$ & $d t$ & $\mathrm{U}$ & $\mathrm{Z}$ & Sig* \\
\hline Magisterio EF & 3,54 &, 718 & 26672,500 &,- 867 &, 386 \\
\hline CAFID - INEF & 3,48 &, 774 & & \\
\hline
\end{tabular}

$\mathrm{U}=\mathrm{U}$ de Mann Whitney. ${ }^{*} p \leq .05$ 
Los maestros de EF son los que profundizan en este tipo de relación y sus ventajas y dan cuenta de cómo los procesos de evaluación formativa y compartida son los que propician en mayor grado este tipo de interacciones.

Considero que es imprescindible que haya una buena relación entre ambas partes para que exista un buen clima de trabajo en el grupo-clase, un adecuado feedback y entre todos se dé un proceso de evaluación compartida y formativa, además de favorecer una mayor confianza y un conocimiento mutuo. (M, 25, EMEF).

La experiencia no resulta ser un factor que determine diferencias significativas ante esta idea generalizada descrita con anterioridad (Tabla 9). A pesar de que los docentes con más de cinco años de experiencia reducen sus respuestas de acuerdo frente a la importancia de este tipo de interacciones para mejorar la evaluación.

Tabla 9. Media, $d t$ y diferencia de medias del ítem 4, por experiencia docente

\begin{tabular}{|l|c|c|c|c|c|}
\hline & $\mathrm{M}$ & $d t$ & Chi-cuadrado & $\mathrm{gl}$ & Sig* \\
\hline Sin experiencia & 3,51 &, 743 & 2,765 & 3 &, 429 \\
\hline $1-2$ años & 3,57 &, 722 & & & \\
\hline 3-4 años & 3,48 &, 814 & & & \\
\hline Más de 5 años & 3,15 &, 987 & & & \\
\hline
\end{tabular}

Prueba de Kruskal-Wallis. ${ }^{*} p \leq .05$

Los datos cualitativos permiten ver el porqué de esta reducción de acuerdo, motivada fundamentalmente por algunos sentimientos de vulnerabilidad del docente en el aula.

La interacción siempre es positiva, pero no debemos olvidar que estas relaciones que se establecen también te hacen vulnerable. A nosotros, los profesores, nos sirve para conocer a nuestros estudiantes y poder adaptarnos a sus ritmos, pero estas relaciones hacen que ellos también tengan unas expectativas hacia nosotros, que nos condicionan a la hora de hacer o de decir en clase. (M, 26, ECAFID).

\subsection{ASPECTOS MEJOR VALORADOS POR LOS ESTUDIANTES PARA LA EVALUACIÓN DE SUS APRENDIZAJES}

\subsubsection{El conocimiento previo del sistema de evaluación}

Según las respuestas ofrecidas al cuestionario, un amplio porcentaje de los participantes $(85,2 \%)$ está de acuerdo o muy de acuerdo con que el conocimiento previo del sistema de evaluación repercute de forma positiva en el aprendizaje del alumno (Tabla 10). Este co- 
nocimiento ayuda al estudiante a prepararse para la prueba de evaluación, le permite autorregular sus tiempos de aprendizaje y le lleva a obtener mejores resultados, como explican los docentes entrevistados.

Puedes prepararte mejor sabiendo qué se te va a evaluar. Los resultados son más positivos porque el proceso es claro desde el principio. (H, 28, EMEF).

Tabla 10. Media y $d t$ de los ítems 5, 6 y 7, aspectos de la evaluación mejor valorados

\begin{tabular}{|l|c|c|}
\hline \multicolumn{1}{|c|}{ Ítem } & $\mathrm{M}$ & \multicolumn{1}{|c|}{$d t$} \\
\hline 5. El conocimiento previo del sistema de evaluación favorece el aprendizaje. & 3,27 &, 861 \\
\hline 6. Las pruebas de evaluación se anunciaron con suficiente antelación. & 3,14 &, 811 \\
\hline 7. Las pruebas de evaluación parten de un acuerdo con el alumnado. & 1,67 & 1,238 \\
\hline
\end{tabular}

Hombres y mujer consideran este aspecto como un hecho destacable en sus experiencias en relación con la evaluación, no existiendo diferencias significativas entre ambos grupos de la muestra (Tabla 11). Además, creen que es necesario informar al estudiante de cómo va a ser evaluado, pues este dato le aporta seguridad y le permite eliminar el nerviosismo que generan las pruebas de evaluación.

Es indispensable que todo alumno sepa de antemano a qué sistema de evaluación debe atenerse, puesto que va a saber a lo que se enfrenta, qué es lo que se le va a pedir y cómo debe de hacerlo. Entiendo que son claves necesarias para un mejor desarrollo del proceso de aprendizaje, ya que promueven en el alumnado una mayor seguridad, tranquilidad y confianza. (M, 25, EMEF).

Tabla 11. Media, $d t$ y diferencia de medias de los ítems 5, 6 y 7 por sexos

\begin{tabular}{|c|c|c|c|c|c|c|}
\hline Ítem & S & M & $d t$ & $\mathrm{U}$ & $\mathrm{Z}$ & $\mathrm{Sig}^{*}$ \\
\hline \multirow{2}{*}{$\begin{array}{l}\text { 5. El conocimiento previo del sistema de } \\
\text { evaluación favorece el aprendizaje. }\end{array}$} & $\mathrm{H}$ & 3,22 & ,0924 & \multirow{2}{*}{25767,500} & \multirow{2}{*}{,- 870} & \multirow{2}{*}{,384 } \\
\hline & M & 3,33 & ,758 & & & \\
\hline \multirow{2}{*}{$\begin{array}{l}\text { 6. Las pruebas de evaluación se anunciaron con } \\
\text { suficiente antelación. }\end{array}$} & $\mathrm{H}$ & 3,13 & ,844 & \multirow{2}{*}{27902,500} & \multirow{2}{*}{,- 020} & \multirow{2}{*}{,984 } \\
\hline & M & 3,15 & ,769 & & & \\
\hline \multirow{2}{*}{$\begin{array}{l}\text { 7. Las pruebas de evaluación parten de un acuerdo } \\
\text { con el alumnado. }\end{array}$} & $\mathrm{H}$ & 1,64 & 1,232 & \multirow{2}{*}{27405,500} & \multirow{2}{*}{,- 587} & \multirow{2}{*}{, 557} \\
\hline & $\mathrm{M}$ & 1,70 & 1,245 & & & \\
\hline
\end{tabular}


Los resultados resultan similares cuando realizamos un análisis con relación a las titulaciones cursadas por los participantes (Tabla 12), siendo los maestros de EF los que dan mayor consideración a este aspecto.

El conocimiento del sistema de evaluación influye al aprendizaje sobremanera, ya que te permite autorregular tu estudio dirigiéndolo a un fin concreto: el formato de prueba de evaluación. Conocer los criterios de evaluación de forma previa, permite autorregular el estudio incidiendo en los aspectos más importantes [...] Por otro lado, para la elaboración de trabajos es vital, pues permiten ajustar todos los apartados a lo que se pide. (M, 24, EMEF).

Tabla 12. Media, $d t$ y diferencia de medias de los ítems 5,6 y 7 por titulación

\begin{tabular}{|c|c|c|c|c|c|c|}
\hline Ítem & $\mathrm{T}$ & M & $d t$ & $\mathrm{U}$ & $\mathrm{Z}$ & Sig* \\
\hline \multirow{2}{*}{$\begin{array}{l}\text { 5. El conocimiento previo del sistema de } \\
\text { evaluación favorece el aprendizaje. }\end{array}$} & A & 3,36 & ,824 & \multirow{2}{*}{23917,000} & \multirow{2}{*}{$-2,153$} & \multirow{2}{*}{,031 } \\
\hline & B & 3,20 & 882 & & & \\
\hline \multirow{2}{*}{$\begin{array}{l}\text { 6. Las pruebas de evaluación se anunciaron } \\
\text { con suficiente antelación. }\end{array}$} & A & 3,17 & ,776 & \multirow{2}{*}{26645,000} & \multirow{2}{*}{,- 693} & \multirow{2}{*}{,489 } \\
\hline & B & 3,11 & ,832 & & & \\
\hline \multirow{2}{*}{$\begin{array}{l}\text { 7. Las pruebas de evaluación parten de un } \\
\text { acuerdo con el alumnado. }\end{array}$} & A & 1,69 & 1,278 & \multirow{2}{*}{27427,000} & \multirow{2}{*}{,- 344} & \multirow{2}{*}{,731 } \\
\hline & B & 1,65 & 1,208 & & & \\
\hline
\end{tabular}

$\mathrm{T}=$ titulación $(\mathrm{A}=$ Magisterio EF. B= CAFID $-\mathrm{INEF}) . \mathrm{U}=\mathrm{U}$ de Mann Whitney. ${ }^{*} p \leq .05$

Los años de experiencia docente tampoco descubren diferencias significativas, como podemos apreciar en la siguiente tabla:

Tabla 13. Media, $d t$ y diferencia de medias de los ítems 5,6 y 7 por experiencia docente

\begin{tabular}{|c|c|c|c|c|c|c|}
\hline Ítem & $\mathrm{E}$ & M & $d t$ & Chi-cuadrado & $\mathrm{gl}$ & Sig* \\
\hline \multirow{4}{*}{$\begin{array}{l}\text { 1. La recogida de información para la } \\
\text { evaluación nos genera tensión y } \\
\text { nerviosismo. }\end{array}$} & A & 3,27 & ,834 & \multirow{4}{*}{3,616} & \multirow{4}{*}{3} & \multirow{4}{*}{,306 } \\
\hline & B & 3,16 & 1,067 & & & \\
\hline & $\mathrm{C}$ & 3,52 &, 750 & & & \\
\hline & D & 3,15 & 0555 & & & \\
\hline \multirow{4}{*}{$\begin{array}{l}\text { 2. La recogida de información para la } \\
\text { evaluación fomenta la motivación para el } \\
\text { aprendizaje. }\end{array}$} & A & 3,15 & ,789 & \multirow{4}{*}{2,290} & \multirow{4}{*}{3} & \multirow{4}{*}{, 514} \\
\hline & B & 3,12 & ,976 & & & \\
\hline & $\mathrm{C}$ & 3,15 & 0671 & & & \\
\hline & D & 2,85 & ,801 & & & \\
\hline
\end{tabular}




\begin{tabular}{|c|c|c|c|c|c|c|}
\hline \multirow{4}{*}{$\begin{array}{l}\text { 3. La evaluación positiva repercute en la } \\
\text { autoestima del/a estudiante. }\end{array}$} & A & 1,68 & 1,243 & \multirow{4}{*}{6,945} & \multirow{4}{*}{3} & \multirow{4}{*}{,074 } \\
\hline & B & 1,70 & 1,314 & & & \\
\hline & $\mathrm{C}$ & 1,10 & 944 & & & \\
\hline & $\mathrm{D}$ & 2,08 & ,862 & & & \\
\hline
\end{tabular}

$\mathrm{E}=$ experiencia $(\mathrm{A}=$ sin experiencia. $\mathrm{B}=1-2$ años. $\mathrm{C}=3-4$ años. $\mathrm{D}=+5$ años $)$. Prueba de Kruskal-Wallis $* p \leq .05$

Pero los datos cualitativos sí nos permiten adentrarnos en el discurso y ver cómo algunos docentes con más de cinco años de ejercicio creen que dar a conocer el sistema de evaluación no es un hecho suficiente si no se consensuan con el estudiante los criterios a adoptar para la evaluación de sus aprendizajes. Algunos participantes explican que si el estudiante no participa en este proceso, el conocimiento previo del sistema de evaluación puede llegar a desmotivarle y abandonar la asignatura.

Puede favorecer el proceso de aprendizaje o al revés, desmotivar a este proceso. (H, 28, EMEF).

\subsubsection{El anuncio de las pruebas de evaluación con suficiente antelación}

Muy relacionado con el ítem anterior, encontramos otro de los aspectos que los participantes reconocen como clave para la mejora de sus aprendizajes y es el anuncio con antelación de las pruebas de evaluación (Tabla 10). Un $82,7 \%$ de la muestra afirma estar de acuerdo o muy de acuerdo con la importancia de este ítem, que también se ve reflejada en los datos cualitativos:

El anuncio de los exámenes o la entrega de trabajos fijada con antelación (en el caso de los exámenes desde principio de curso) facilitan la planificación del estudio. (M, 24, EMEF).

El análisis entre los grupos de maestros de EF y los profesores de CAFID-INEF no presenta datos significativos que nos permitan diferenciar respuestas contratadas. Los participantes parecen haber vivido durante su FIP el anuncio de las pruebas de evaluación con suficiente anticipación y explican que si este hecho no se hubiera producido, hubieran tenido mayores índices de ansiedad y sus calificaciones habrían sido peores.

Durante mi etapa como estudiante de Grado creo que todos los exámenes fueron fijados con antelación. No tuvimos "pruebas sorpresas" que sin duda hubieran generado ansiedad a la hora de llevar al día la materia. (M, 24, EMEF).

Tampoco se presenta una diferencia significativa cuando valoramos los datos en función de la experiencia (Tabla 13). Los docentes creen, como explican en las entrevistas, que el anuncio de las pruebas de evaluación con suficiente tiempo de antelación repercute de forma positiva en la adquisición de conocimientos, mejora la predisposición al aprendizaje 
de los contenidos y las competencias de la materia y favorece una calificación positiva.

Resulta interesante destacar que la mayoría de docentes siguieron avisando a sus estudiantes, en los primeros años de su carrera profesional, de las pruebas de evaluación. No obstante, algunos admiten que en ocasiones las exigencias del centro no les permitieron cumplir con este objetivo que ellos consideran valioso para la mejora del aprendizaje y de la calificación.

\subsubsection{El acuerdo de las pruebas de evaluación entre docente y estudiantes}

Con relación a los apartados de análisis anteriores, uno de los aspectos que destacan los participantes como relevante para la mejora de los aprendizajes de los estudiantes es el acuerdo de las pruebas de evaluación entre docentes y estudiantes.

Si queremos que el alumnado se sienta parte de su aprendizaje, tenemos que hacerles parte de todo el proceso y en ese proceso se encuentra la toma de decisiones sobre la evaluación. Se ha de llegar a un acuerdo entre profesorado y alumnado de forma consensuada y justificada. (H, 28, EMEF).

Resulta significativo apreciar que, a pesar de considerar este aspecto como uno de los más importantes para la evaluación de los aprendizajes, tanto las respuestas de los participantes en los cuestionarios (Tabla 10) como las aportadas en las entrevistas, dan muestras de que los protagonistas del estudio no han vivido en su FIP un acuerdo en las pruebas de evaluación con sus profesores.

No recuerdo haber vivido ningún acuerdo en las pruebas de evaluación durante mi formación inicial. Los días de los exámenes estaban pautados en el calendario académico oficial, desde que comenzó el curso y también el tiempo en el que debían ser realizados... además, era el profesor el que establecía las reglas de los materiales con los que podíamos contar y el que calificaba las pruebas. Sólo en algunas asignaturas pudimos participar en la evaluación de los trabajos de nuestros compañeros, pero fueron casos puntuales. (M, 30, EMEF).

No encontramos diferencias significativas ni entre sexos (Tabla 11) ni entre titulaciones (Tabla 12), y los datos cualitativos nos llevan a señalar que sigue existiendo una fuerte tendencia en Educación Superior hacia procesos de evaluación tradicional. Los participantes hacen una crítica en sus entrevistas a la falta de consideración del profesorado hacia los estudiantes para acordar los contenidos de las pruebas de evaluación y consideran injusto que el alumnado sea apartado de este consenso.

Si el alumnado es protagonista de todo este proceso, tiene que tener "opinión y voto" en cuanto a las pruebas de evaluación. Cuando eres partícipe de algo, asumes la responsabilidad en mayor medida que si te viene impuesto, por tanto ves más justa y coherente la decisión final. (H, 38, EMEF).

La experiencia tampoco parece ser un factor condicionante en las encuestas (Tabla 13), a pesar de que en las entrevistas sí podemos advertir algunos datos que resultan de interés. Hemos apreciado que aquellos docentes que llevan poco tiempo en ejercicio, afirman haber 
empezado a vivir en los últimos años de su FIP algunos acuerdos con los profesores en relación con los trabajos que tenían que entregar y los contenidos de los que iban a ser examinados. Los docentes con más de cinco años de experiencia explican que apenas vivieron este tipo de situaciones, pero su participación en algunos cursos y proyectos de formación permanente, vinculados con la evaluación formativa, les han llevado a aplicar este tipo de experiencias en el aula.

Empecé reproduciendo la evaluación que había vivido, pero a partir de un curso de evaluación formativa, que nos ofreció el centro a los maestros, me empecé a dar cuenta de que había otra forma de evaluar que no era la que yo había experimentado [...] Al principio pensaba que cuando la llevara al aula no daría resultado, porque los alumnos eran pequeños y no íbamos a ser capaces de llegar a acuerdos, pero con el tiempo me he dado cuenta de que esta es una forma de que aprendan, de que participen, de que mejoren. (H, 30, ECAFID).

\section{DISCUSIÓN DE RESULTADOS}

A la luz de los resultados recogidos y su análisis, procedemos a la discusión de los resultados que nos han ayudado a conocer cómo los docentes de EF han vivido la evaluación durante su FIP. Haremos hincapié en cada una de las categorías de análisis para perfilar el objetivo general del estudio.

Desde la primera categoría de análisis, en la que se trataba de comprobar los sentimientos y emociones que produce la evaluación en los docentes de EF, hemos podido comprobar que los participantes no muestran un grado elevado de tensión hacia la recogida de información para la evaluación $(2,52)$. Este dato discrepa de investigaciones como las de Trillo y Porto (1999) o Ricoy y Fernández-Rodríguez (2013) en el que un alto porcentaje de estudiantes manifestaba que las pruebas de evaluación le producían ansiedad y sensaciones negativas y nos permite ver que la forma de entender la evaluación en Educación Superior está cambiando, gracias a la inclusión de los estudiantes en los procesos de evaluación (Hortigüela, Pérez-Pueyo \& Salicetti, 2016b; López-Pastor, Castejón \& Pérez-Pueyo, 2012).

Por sexos, vemos que las docentes en EF presentan mayores niveles de ansiedad y tensión hacia la evaluación que los hombres. Este dato coincide con estudios como el de Ayora (1993), pues las mujeres afirman que se sienten más observadas en las pruebas físicas, sienten temor a las exposiciones orales y su ansiedad es mayor ante un examen. Los docentes masculinos no hacen visibles sus sentimientos ante este hecho, a pesar de mostrar niveles semejantes de ansiedad. Este silencio puede deberse a que los participantes varones no quieren manifestar algunos sentimientos que les puedan hacer sentirse vulnerables o contradigan los modelos masculinos hegemónicos aprendidos en su formación previa, presentado una barrera a la expresión de sus experiencias y a su desarrollo personal (Mellado, Luengo \& Montaña, 2016).

La experiencia también resulta ser un dato clave en el análisis, pues a partir del tercer y el cuarto año de ejercicio, los docentes sienten menor ansiedad ante las pruebas de evaluación. Posiblemente, este hecho se deba a que, en este periodo de ejercicio, ya han superado una primera fase en la que les preocupaban algunos aspectos como el control del 
aula, el desarrollo de actividades o la programación de sus clases, y a partir de ese momento se preocupen por aspectos más didácticos y educativos.

Además, en el análisis cualitativo hemos podido comprobar que los instrumentos clave para evaluar los aprendizajes de los docentes siguen siendo las pruebas objetivas, los exámenes escritos y los trabajos, como explican los protagonistas, y que también se hacen visibles en trabajos como los de Martínez, Castejón y Santos (2012) o Gutiérrez, Pérez Pueyo y Pérez (2013). Este tipo de instrumentos genera mayor tensión y ansiedad entre los docentes de EF, que otros como los cuadernos, diario o los casos prácticos (Trillo \& Porto, 1999), pruebas que permiten que el estudiante exprese sus miedos, aprenda de ellos y mejore su aprendizaje.

Desde la subcategoría que analiza si la evaluación motiva el aprendizaje del estudiante, nuestro estudio coincide con la investigación de Trillo y Porto (1999), pues los docentes de EF no se han sentido partícipes de esta evaluación y no encuentran esa relación entre evaluación y aprendizaje en sus vivencias en la FIP. Experiencias como las de LópezPastor et al., (2012), o Ponn et al., 2009, en las que los estudiantes participan activamente en los procesos de evaluación, nos enseñan cómo, cuando existe esta participación, los estudiantes sí perciben las pruebas de evaluación como momentos de aprendizaje.

Con relación a cómo la evaluación positiva repercute en la autoestima del estudiante, los participantes expresan que esta repercusión es alta $(3,46)$, coincidiendo con trabajos como los de Ricoy y Fernández-Rodríguez (2013) o Trillo y Porto (1999). Los protagonistas sienten una mejora de su motivación y de su autoconcepto cuando reciben buenas calificaciones, pero el caso contrario les hace bajar su rendimiento y en algunos casos cuestionarse la profesión docente. Estudios como los de Doménech (2011) dejan claras las repercusiones de la evaluación en la autoestima del estudiante, por ello invitan a los profesores universitarios a ser conscientes de esta realidad y a presentar un modelo de evaluación basado en la implicación del alumno, que permita el desarrollo de sus conocimientos, sus habilidades y sus actitudes.

Profundizando en la discusión de la segunda categoría de análisis, apreciamos que los docentes de EF consideran que las interacciones entre profesores y estudiantes resultan cruciales para la mejora del proceso de evaluación $(3,51)$, pero afirman que en sus experiencias universitarias no han vivido estas relaciones en muchas asignaturas, coincidiendo este dato con la investigación de Trillo y Porto (1999). Las interacciones son fundamentales para la mejora del proceso de enseñanza-aprendizaje (Escobar, 2015) y en ellas se hacen patentes algunos aspectos como las emociones, los sentimientos o la autoestima que tiene el estudiante, factores cruciales que no deben ser olvidados en la evaluación. La comunicación entre profesores y estudiantes es uno de los aspectos mejor valorados en las entrevistas realizadas a los docentes de EF, pues la comunicación favorece la progresión del aprendizaje del alumno, motiva el desarrollo de su sentido crítico y reflexivo así como la mejora de sus habilidades y destrezas en la formación (Granja, 2013). Las docentes de EF dan mayor valor a estas interacciones, afirmando que la empatía, el apoyo y la ayuda por parte del maestro mejora el rendimiento académico del estudiante y su aprendizaje.

Analizando la tercera categoría marcada, que buscaba conocer los aspectos mejor valorados por los estudiantes para la evaluación de sus aprendizajes, hemos constatado que los participantes muestran un acuerdo mayoritario en los beneficios que produce la participación activa de los estudiantes en el proceso de evaluación, como también muestran 
los estudios de Hortigüela et al. (2015a) y Rodríguez et al. (2013). El conocimiento previo del sistema de evaluación, el anuncio con antelación de las pruebas de evaluación y el acuerdo de las pruebas de evaluación entre docentes y estudiantes, constituyen los aspectos mejor valorados por los docentes para la correcta evaluación de los aprendizajes.

Tanto el conocimiento previo del sistema de evaluación $(3,27)$ como el anuncio con antelación de las pruebas de evaluación $(3,14)$, parecen haber sido aspectos vividos por los docentes en EF de forma recurrente en las asignaturas de sus carreras, y ellos mismos reproducen estas conductas en su actividad ordinaria. No ocurre lo mismo con su participación en el proceso de evaluación, aspecto que los participantes reconocen no haber vivido a lo largo de su formación $(1,67)$. Este dato coincide con el de investigaciones como las de Trillo y Porto (2009). A pesar de no haber vivido esta experiencia, los participantes afirman que, si este acuerdo se produjera, aumentarían sus niveles de formación y desarrollo profesional, coincidiendo con los datos de trabajos como los de Brown y Glasner (2007), Ibarra y Rodríguez (2014), Martínez et al. (2012), Martínez et al. (2017), o Ruiz, Ruiz y Ureña (2013). Los docentes no empiezan a favorecer este tipo de acuerdos hasta que no llevan más de cinco años en ejercicio. Posiblemente, no deciden hacerlo hasta que no consolidan su desarrollo profesional.

\section{CONCLUSIONES}

El estudio presentado muestra algunos cambios producidos en los últimos años, en el proceso de evaluación de la FIP. Si bien, la evaluación sigue generando sentimientos de ansiedad y temor para los estudiantes y las pruebas de evaluación no son vistas como las principales motivaciones para el aprendizaje, la implicación de los estudiantes en los procesos de evaluación favorece que estos tengan una vivencia positiva de este hecho y se impliquen en la asunción de responsabilidades. Dicha implicación repercute positivamente en su proceso de aprendizaje y también en el rendimiento académico, lo que posibilita mejores calificaciones y, por ende, motiva su autoestima.

La interacción entre profesores y estudiantes es un aspecto de suma importancia para los docentes de EF. El diálogo, la empatía y el conocimiento de los sentimientos y las necesidades que el estudiante tiene en su proceso formativo mejoran los resultados de la evaluación.

El conocimiento previo del sistema de evaluación, el anuncio con antelación de las pruebas de evaluación y el acuerdo de las pruebas de evaluación entre docentes y estudiantes son tres aspectos importantes para los participantes y repercuten de forma significativa en sus vivencias en relación con la evaluación. Estos aspectos les ayudan a mejorar su autocontrol, a optimizar sus actitudes hacia las pruebas de evaluación y, por tanto, les ayudan también a la mejora de su aprendizaje.

Por último, nos gustaría señalar algunas limitaciones del estudio, así como futuras líneas de investigación. Entre las primeras, cabe destacar el número de participantes de la muestra (especialmente en la parte cualitativa) y la reducción geográfica del estudio. Además, con el objetivo de profundizar en los datos, podría ser interesante dividir a la muestra que marca este estudio entre docentes recién egresados y aquellos que llevan titulados más de cinco años, con el objetivo de establecer otro tipo de comparaciones entre grupos o conocer las diferencias en relación con las diferentes localidades. Estas carencias 
nos llevan a presentar posibles líneas de trabajo a partir de este estudio, entre las que destacamos realizar un análisis exclusivamente cualitativo de las vivencias de los docentes con relación a la evaluación (especialmente vinculadas con los sentimientos y emociones que esta produce, pues este resulta ser el aspecto menos tratado hasta el momento), que permitan penetrar en el conocimiento subjetivo que el estudiante tiene del proceso de evaluación vivido. También podría ser recomendable hacer un estudio retrospectivo de cómo han cambiado las vivencias que los estudiantes tienen en relación con la evaluación en este siglo o dar a conocer los aspectos peor valorados en la FIP por los estudiantes en los procesos de evaluación.

\section{REFERENCIAS BIBLIOGRÁFICAS}

Angelini, M.L. (2016). Estudio sobre la evaluación formativa y compartida en la formación docente en inglés. Actualidades investigativas en Educación, 16(1), 1-21.

Ayora, A. (1993). Ansiedad en situaciones de evaluación o examen en estudiantes secundarios de la ciudad de Loja (Ecuador). Revista Latinoamericana de Psicología, 25(3), 425- 431.

Brown, S., \& Glasner, A. (2003). Evaluar en la universidad. Problemas y nuevos enfoques. Madrid: Narcea.

Brown, S., \& Glasner, A. (Eds.). (2007). Evaluar en la Universidad. Problemas y nuevos enfoques (2a ed.). Madrid: Narcea.

Buskist, W., Sikorski, J., Buckley, T., \& Saville, B. K. (2002). Elements of master teaching. In S. F. Davis \& W. Buskist (Eds.), The teaching of psychology: Essays in honor of Wilbert J. McKeachie and Charles L. Brewer (pp. 27-39). Mahwah, NJ: Lawrence Erlbaum Associates.

Casillas, S. (2006). Percepciones de alumnos y profesores sobre el "buen" docente universitario. Papeles salmantinos de Educación, 7, 271-282.

Doménech, F. (2011). Evaluar e investigar en la situación educativa universitaria: un nuevo enfoque desde el Espacio Europeo de Educación Superior. Castelló de la Plana: Publicacions de la Universitat Jaume I.

Escobar, M.B. (2015). Influencia de la interacción alumno docente en el proceso de enseñanza aprendizaje. Paakat, 8(5), 15-23.

Flórez, J. (1999). Evaluación de la calidad de la docencia. León: Universidad de León.

Gargallo, B., Sánchez, F.J., Ros, C., \& Ferreras, A. (2010). Estilos docentes de los profesores universitarios: la percepción de los alumnos de los buenos profesores. Revista Iberoamericana de Educación, 51(4), 1681-5653.

Granja, C. (2013). Caracterización de la comunicación pedagógica en la interacción docente-alumno. Investigación, Enfermería y Desarrollo, 15(1), 65-93.

Gutiérrez, C., Pérez Pueyo, A., \& Pérez, M. (2013). Percepciones de profesores, alumnos y egresados sobre los sistemas de evaluación en estudios universitarios de formación del profesorado de educación física. Ágora para la educación física y el deporte, 2(15), 130-151.

Hamodi, C., \& López Pastor, A.T. (2012). La evaluación formativa y compartida en la Formación Inicial del Profesorado desde la perspectiva del alumnado y los egresados. Psychology, Society \& Education, 4(1), 103-116.

Herranz, M., \& López Pastor, V.M. (2017). Perspectiva del alumno sobre su participación en los procesos de evaluación formativa y compartida en Educación Física en Primaria: un estudio de caso. EmasF, 48, 27-48.

Hortigüela, D., Abella, V., \& Pérez-Pueyo, A. (2015a). Percepciones del alumnado sobre la evaluación formativa: contraste de grupos de inicio y final de carrera. Revista de Docencia Universitaria, 13(3), 13-32. 
Hortigüela, D., Pérez-Pueyo, A., \& Salicetti, A. (2015b). ¿Cómo percibe el alumnado universitario de educación física la evaluación recibida? Contraste de dos metodologías diferentes. RETOS, 28, 66-70.

Hortigüela, D., \& Pérez-Pueyo, A. (2016). Percepción del alumnado de las clases de Educación Física en relación con otras asignaturas. Apunts, 123, 44-52.

Ibarra, M.S., \& Rodríguez, G. (2014). Modalidades participativas de evaluación: Un análisis de la percepción del profesorado y de los estudiantes universitarios. Revista de Investigación Educativa, 32(2), 339-361. doi:10.6018/rie.32.2.172941

López Pastor, V.M. (2004). Evaluación, calificación, credencialismo y formación inicial del profesorado: efectos y patologías generadas en la enseñanza universitaria. Revista Interuniversitaria de Formación del Profesorado, 51, 221-232.

López Pastor, V. M. (2008). Implementing a Formative and Shared Assessment System in Higher Education Teaching. European Journal of Teacher Education, 31(3), 293-311.

López Pastor, V.M. (2009). La evaluación formativa y compartida en docencia universitaria. Madrid: Narcea.

López Pastor, V.M., Castejón, J., \& Pérez Pueyo, (2012). ¿Implicar al alumnado en la evaluación en la formación inicial del profesorado? Un estudio de caso de evaluación entre iguales de un examen. Multidisciplinary Journal of Educational Research, 2, 77-201.

López Pastor, V. M., \& Palacios, A. (2012). Percepción de los futuros docentes sobre los sistemas de evaluación de sus aprendizajes. Revista Teoría de la Educación: Educación y Cultura en la Sociedad de la Información, 13(3), 317-341.

Martínez, L., Castejón, J., \& Santos, M.L. (2012). Diferentes percepciones sobre evaluación formativa entre profesorado y alumnado en formación inicial en educación física. REIFOP, 15(4), 57-67.

Martínez, L.F., Santos, M.L., \& Castejón, F.J. (2017). Percepciones de alumnado y profesorado en Educación Superior sobre la evaluación en formación inicial en educación física, RETOS, 32, 76-81.

Mellado, L., Luengo, M.R., \& Montaña, J.L. (2016). Las metáforas emocionales de profesores en formación de Infantil, Primaria y Psicopedagogía. Enseñanza \& Teaching, 34(1), 179-195.

Nieto, S. (2000). El discurso del profesorado universitario sobre la evaluación del aprendizaje de los alumnos como estrategia de innovación y cambio profesional: exposición y análisis de una experiencia. Revista de Educación, 322, 305-324.

Palacios, A., \& López Pastor, V. M. (2013). Haz lo que yo digo pero no lo que yo hago: sistemas de evaluación del alumnado en la formación inicial del profesorado. Revista de Educación, 361, 279-305.

Ponn, W., McNaught, C., Lam, P., \& Kwan, S. (2009). Improving assessment methods in university science education with negotiated self- and peer-assessment. Assessment in Education: Principles, Policy \& Practice, 3, 331-346.

Porto, M. (2009). Evaluación de estudiantes en la universidad de Santiago de Compostela: percepciones de los propios alumnos. Revista de Docencia Universitaria, 3. Recuperado de http://red-u.net/redu/files/journals/1/articles/99/public/99-85-2-PB.pdf

Ricoy, M.C., \& Fernández Rodríguez, J. (2013). La percepción que tienen los estudiantes universitarios sobre la evaluación: un estudio de caso. Educación XXI, 16(2), 321-341.

Rodríguez, G., Ibarra, M.S., \& García, E. (2013). Autoevaluación, evaluación entre iguales y coevaluación: conceptualización y práctica en las universidades españolas. Revista de Investigación en Educación, 11(2), 198-210.

Ruiz, J.R., Ruiz, E., \& Ureña, N. (2013). La evaluación en la formación inicial del profesorado: que creemos hacer y que perciben los alumnos. Cultura, Ciencia y Deporte, 9(8), 17-29.

Silva, I., \& López Pastor, V. (2015). ¿Cómo vive el alumnado la evaluación en formación inicial del profesorado? Revista d'innovació educativa, 14, 90-100. doi:10.7203/attic.14.4171

Tejedor, F. J. (Dir.). (1998). Las estrategias utilizadas por los profesores universitarios para la 
evaluación del aprendizaje de los alumnos. Madrid: CIDE-Informe.

Trillo, F. (2005). La evaluación de los estudiantes universitarios. El caso de la Universidad de Santiago de Compostela. Santiago de Compostela: Universidad de Santiago de Compostela.

Trillo, F., \& Porto, M. (1999). La percepción de los estudiantes sobre su evaluación en la universidad. Un estudio en la Facultad de Ciencias de la Educación. Innovación Educativa, 9, 55-75.

Zabalza, M. (2003). Diseño curricular en la universidad. Competencias del docente universitario. Madrid: Narcea. 
ANEXO 1. Cuestionario utilizado para la recogida de datos

\begin{tabular}{|c|c|c|c|c|c|}
\hline $\begin{array}{l}\text { Grado de acuerdo con las siguientes } \\
\text { afirmaciones }\end{array}$ & $\begin{array}{l}\text { Nada de } \\
\text { acuerdo }\end{array}$ & $\begin{array}{l}\text { Poco de } \\
\text { acuerdo }\end{array}$ & $\begin{array}{c}\text { Ni acuerdo ni } \\
\text { desacuerdo }\end{array}$ & $\begin{array}{c}\text { De } \\
\text { acuerdo }\end{array}$ & $\begin{array}{l}\text { Muy de } \\
\text { acuerdo }\end{array}$ \\
\hline $\begin{array}{l}\text { 1. La interacción entre profesores y } \\
\text { estudiantes favorece el proceso de } \\
\text { evaluación. }\end{array}$ & 0 & 1 & 2 & 3 & 4 \\
\hline $\begin{array}{l}\text { 2. La realización de las pruebas de } \\
\text { evaluación se anunció con suficiente } \\
\text { antelación. }\end{array}$ & 0 & 1 & 2 & 3 & 4 \\
\hline $\begin{array}{l}\text { 3. Las pruebas de evaluación partieron de } \\
\text { un acuerdo con el alumnado. }\end{array}$ & 0 & 1 & 2 & 3 & 4 \\
\hline $\begin{array}{l}\text { 4. La recogida de información para la } \\
\text { evaluación nos genera estrés y } \\
\text { nerviosismo. }\end{array}$ & 0 & 1 & 2 & 3 & 4 \\
\hline $\begin{array}{l}\text { 5. La recogida de información para la } \\
\text { evaluación fomenta la motivación para el } \\
\text { aprendizaje. }\end{array}$ & 0 & 1 & 2 & 3 & 4 \\
\hline $\begin{array}{l}\text { 6. La evaluación positiva repercute en la } \\
\text { autoestima del estudiante. }\end{array}$ & 0 & 1 & 2 & 3 & 4 \\
\hline $\begin{array}{l}\text { 7. El conocimiento previo del sistema de } \\
\text { evaluación favorece nuestro proceso de } \\
\text { aprendizaje. }\end{array}$ & 0 & 1 & 2 & 3 & 4 \\
\hline
\end{tabular}

\section{ANEXO 2. GuiOn de la entrevista}

1. ¿Crees que la interacción entre profesores y estudiantes favorece el proceso de evaluación? ¿Por qué?

2. ¿Cómo influyo el anuncio con antelación de las pruebas de evaluación en tus resultados?

3. ¿Consideras que las pruebas de evaluación deben partir de un acuerdo con el alumnado? ¿Por qué?

4. ¿Cómo viviste los momentos de recogida de información para tu evaluación?

5. ¿Piensas que la recogida de información para la evaluación fomenta la motivación para el aprendizaje? ¿Por qué?

6. Cuando recibiste una evaluación positiva ¿Cómo repercutió esta calificación en tu autoestima?

7. ¿Consideras que el conocimiento previo del sistema de evaluación favorece el proceso de aprendizaje del/a estudiante? ¿Por qué? 
